\title{
Hairpin Telomere Resolvases
}

Kerri Kobryn ${ }^{1}$ and George Chaconas ${ }^{2}$

${ }^{1}$ Department of Microbiology and Immunology, College of Medicine, University of Saskatchewan, Saskatoon, SK S7N 5E5, Canada: email: kerri.kobryn@usask.ca;

${ }^{2}$ Department of Biochemistry \& Molecular Biology and Department of Microbiology, Immunology \& Infectious Diseases, Snyder Institute, The University of Calgary, Calgary, AB T2N 4N1, Canada; email: chaconas@ucalgary.ca 


\begin{abstract}
Covalently closed hairpin ends, also known as hairpin telomeres, provide an unusual solution to the end replication problem. The hairpin telomeres are generated from replication intermediates by a process known as telomere resolution. This is a DNA breakage and reunion reaction promoted by hairpin telomere resolvases (also referred to as protelomerases) found in a limited number of phage and bacteria. The reaction promoted by these enzymes is a chemically isoenergetic two-step transesterification without a requirement for divalent metal ions or high-energy cofactors and uses an active site and mechanism similar to that for type IB topoisomerases and tyrosine recombinases. The small number of unrelated telomere resolvases characterized to date all contain a central, catalytic core domain with the active site, but in addition carry variable $\mathrm{C}$ - and $\mathrm{N}$-terminal domains with different functions. Similarities and differences in the structure and function of the telomere resolvases are discussed. Of particular interest are the properties of the Borrelia telomere resolvases, which have been studied most extensively at the biochemical level and appear to play a role in shaping the unusual segmented genomes in these organisms and, perhaps, to play a role in recombinational events.
\end{abstract}




\section{Hairpin telomere resolvases}

Hairpin telomere resolvases (also known as protelomerases) have emerged as a unique solution to the end replication problem $(1,2)$. These enzymes promote the formation of covalently closed hairpin ends on linear DNA molecules in some phage (35), bacterial plasmids and bacterial chromosomes (6-9). Telomere resolvases are mechanistically related to tyrosine recombinases and type IB topoisomerases and are also believed to play a role in the genome plasticity that characterizes Borrelia species. Fig. 1 shows the reaction pathway for replication of linear DNA molecules with covalently closed hairpin telomeres. Duplication of the DNA molecule results in replicated telomeres ( $r T e l$, also referred to as dimer junctions) that are recognized and processed in a DNA breakage and reunion reaction promoted by a hairpin telomere resolvase. The reaction products are covalently closed hairpin telomeres at both ends of linear monomeric DNA molecules. At this writing telomere resolvases have been purified from three phage and seven bacterial species: E. coli phage N15 (3), Klebsiella oxytoca phage $\varphi$ KO2, Yersinia enterocolitica phage PY54 (5), Agrobacterium tumefaciens (8), the Lyme spirochete Borrelia burgdorferi (6) the relapsing fever borreliae B. hermsii, $B$. parkeri, B. recurrentis, B. turicatae, and the avian spirochete $B$. anserina (7). The $B$. burgdorferi enzyme, ResT (Resolvase of Ielomeres) has been the most extensively studied at the biochemical level $(6,7,10-23)$ and is the primary focus of this review, with properties of the other enzymes noted $(3-5,8,24)$. Structural studies of the Klebsiella phage $\varphi \mathrm{KO} 2$ (25) and the Agrobacterium (26) resolvases have been reported and have shed additional light on reaction mechanisms and on differences between the resolvases from different organisms. 


\section{Reaction mechanism - similarity to type IB topoisomerases and tyrosine recombinases}

ResT and other telomere resolvases/protelomerases constitute a new class of DNA breakage and rejoining enzymes since they catalyze a unique reaction in which a replicated telomere $(r T e /)$ substrate is cleaved 6 base pairs apart $(4,6,8)$ on opposite strands at the centre of the substrate, giving rise to covalently closed hairpin telomeres after the 6 nt 5'-overhangs have been folded back to effect strand rejoining.

Despite the unique hairpin products produced by the telomere resolution reaction the reaction has key similarities to the reactions catalyzed by the type IB topoisomerase and tyrosine recombinase enzyme families. A principle similarity lies in the fact that the DNA breakage and reunion reactions proceed as a chemically isoenergetic two-step transesterification without a requirement for divalent metal ions or high-energy cofactors. This is made possible by a shared mode of phosphoryl transfer in which DNA cleavage is effected by attack on the scissile phosphate(s) by an active site tyrosine accompanied by formation of a transient protein-DNA covalent complex that stores the phosphodiester bond energy; the cleaved DNA strand(s) are resealed by attack on the 3'phosphotyrosine linkage by the 5'-OH group of the cleaved strand(s) to form the final products releasing the enzyme.

The products of the reactions of these enzyme families vary by virtue of differences in the number of DNA strands cleaved and in the identity of the strands that are rejoined. The type IB topoisomerases cleave a single DNA strand that is rejoined after duplex rotation or strand passage to change levels of DNA supercoiling; the type IB topoisomerases act as monomers (27). Tyrosine recombinases cleave four DNA strands 
in two reaction steps and rejoin the cleaved strands to new partners to produce recombinant products. Consequently, the tyrosine recombinases act as tetramers that form a synaptic complex of the reaction sites; strand exchange occurs in the context of the synaptic complex $(28,29)$. The reaction of telomere resolvases lies midway in this spectrum; two DNA strands are cleaved and after strand foldback the 5'-overhanging strands are rejoined to the opposite strand to form two hairpin telomeres. Because of these differences telomere resolvases act as dimers on a single $r T e /$ reaction site $(22$, $25,26)$. In some ways telomere resolution may be thought of as a stripped down sitespecific recombination reaction that occurs with only one reaction site rather than two (4, 14).

Similar to the tyrosine recombinases it is known that ResT requires inter-protomer communication to initiate reaction chemistry. ResT is inactive on a half-site embedded in a plasmid and requires oligonucleotide half-sites to assemble into a 'cross-axis complex' in which a dimer of ResT brings the two half-sites into an arrangement that mimics the structure of the bona fide rTel substrate (Fig. 2) for reaction chemistry to proceed (14, 16). Formation of the cross-axis complex requires conformational changes in ResT to unmask the telomere binding determinants in the catalytic core and/or the C-terminal domain of ResT, which are partially occluded by the N-terminal domain of the protein (18). Unexpectedly, positive DNA supercoiling helps to overcome this barrier, promoting cooperative binding of ResT to rTels and subsequent cross-axis complex formation (16); negative supercoiling inhibits the reaction. The positive DNA supercoiling likely promotes positive DNA rotation in the centre of the $r T e /$ to facilitate the cooperative interactions required for cross-axis complex formation to occur. 
Tyrosine recombinases have a strict temporal order and a half-of-sites activity scheme in which only one protomer in the dimer bound to each reaction site can be active at a time, such that the reactions transit through the Holliday junction $(\mathrm{HJ})$ intermediate without producing double-strand breaks in the substrate DNAs (30-32). Tyrosine recombinases will usually regenerate substrate if they cannot complete a reaction cycle $(33,34)$. In contrast to this, ResT activity on the two sides of the $r T e /$ must be simultaneous, or nearly so, in order that the $5^{\prime}-\mathrm{OH}$ nucleophiles for the second pair of transesterifications (hairpin formation) are both available at the same time (14). Because of these features it was of interest to know whether the reaction chemistry of DNA cleavage and strand transfer in telomere resolution and in the reverse reaction of telomere fusion were concerted on the two halves of the substrate, linking reaction completion on the two sides, or whether the reaction chemistry on the two half-sites could be uncoupled from each other. Using substrates modified so as to inhibit either DNA cleavage or DNA strand transfer on one half-site but not the other, it was determined that execution of both chemical steps on the two halves of the reaction substrate can be uncoupled from each other (14). This combination of simultaneous action on the two halves of the substrate with the freedom to uncouple the completion of the DNA cleavage or strand transfer reactions on the two sides of the substrate was somewhat surprising since it implied that in certain conditions a hairpin telomere might be formed on one side of the substrate at the expense of a ResT-capped double-strand break on the other half of the rTel. 


\section{A tyrosine recombinase-like active site}

A weak similarity of putative telomere resolvases to tyrosine recombinases was noted in early sequence alignments $(6,35)$. A detailed study of the putative active site residues for ResT based on these alignments and on alignments among confirmed or suspected telomere resolvases was made $(7,13)$. Similar to type IB topoisomerases and tyrosine recombinases, ResT can cleave substrates modified at the scissile phosphates with 5'-bridging phosphorothiolates but cannot use the liberated 5'-sulphydryl groups as nucleophiles for the subsequent strand transfer by virtue of the lower pKa of sulphydryl compared to hydroxyl groups $(33,36-38)$. These modified substrates can therefore be used as suicide substrates to accumulate the covalent ResT-DNA complex. ResT cleavage of such a suicide substrate, followed by affinity purification of tryptic peptides attached to the DNA, allowed the identification of the active site tyrosine nucleophile as Y335 (see Fig. 3) by tandem mass spectroscopy (13).

Tyrosine recombinases use a constellation of additional conserved active site residues $(\mathrm{RKHRH} / \mathrm{W})$ thought to be involved in transition state stabilization and in general acid/base chemistry for the protonation/deprotonation of leaving groups and nucleophiles, respectively $(29,39-41)$. Alignments imply an active site composition of RKYRH for ResT in addition to the nucleophilic tyrosine. Examination of mutants of ResT in the corresponding positions revealed that mutation of most of these residues produce a resolvase that can still bind DNA with normal affinity but have dramatically reduced telomere resolution proficiency. Another interesting feature for ResT is that the final residue in the putative active site is a highly conserved histidine but was found to be dispensable for the reaction (13). This is reminiscent of the situation in the Flp 
recombinase in which this residue is a tryptophan that plays a mainly structural rather than a catalytic role (40). Moreover, no evidence was obtained for any of the expected active site residues or additional conserved basic residues queried to play a role in leaving group protonation (13). This was investigated using the approach of rescue of DNA cleavage defects of putative general acid mutants by reaction with substrates containing a 5'-bridging phosphorothiolate substitution for the scissile phosphate $(13,33$, 36-38). This result could indicate water-mediated chemistry (42) or an additional critical role for the suspected general acid residue (K224) in the reaction, precluding rescue solely by lowering the pKa of the leaving group.

There are now crystal structures of two hairpin telomere resolvases from a Klebsiella oxytoca phage with a linear plasmid prophage (TelK) and from Agrobacterium tumefaciens, a bacterium that possesses a linear as well as a circular chromosome (TelA). The structures of TelK and TelA were solved in complex with cleaved $r T e /$ and hairpin product, respectively. The active site of TelK is present as a mimic of the transition state of the DNA cleavage step and shows an active site that is a hybrid of that found in type IB topoisomerases and tyrosine recombinases with the RKHRH constellation being composed of an RKKRH constellation instead. The structure of the active site supports the proposed two-step transesterification mechanism inferred for telomere resolution (25). The active site of TelA, obtained as a transition state mimic of strand transfer/ligation, shows the expected arrangement of active site residues with the third active site residue represented by a tyrosine, as it is in $\operatorname{Res} T(13,26)$. This position has been implicated in studies on Flp as important for protonation of the tyrosine leaving group in the second transesterification (41). Protonation of tyrosine is less dependent 
upon enzyme mediation than that of the 5'-OH leaving group; this is consistent with the less severe defect of the ResT (Y293A) mutant in telomere resolution than mutation of the other active site residues (13).

\section{Telomere substrates}

A ubiquitous feature of the substrates for hairpin telomere resolvases $(3-5,8,11$, 24,43 ) is their 180 -degree rotational symmetry (see L'L and RR', Fig. 1). The reaction is concerted in that both halves of the substrate are required before reaction chemistry will ensue $(3,14,16,24)$. Negative supercoiling of the substrate is a potent inhibitor of ResT $(6,16)$ and the reaction is dramatically stimulated by positive supercoiling, discussed further in a later section (16). In contrast, TelA can process a negatively supercoiled substrate, although not as efficiently as one that is relaxed (8).

An interesting feature of Borrelia species is their segmented genomes with a linear plasmid and multiple linear plasmids. The prototype B. burgdorferi strain B31 contains almost two dozen replicons, most of which are linear $(44,45)$. This strain contains 19 unique but related hairpin telomeres (Fig. 4) necessitating a relaxed ability for substrate utilization by ResT that is not found in the other characterized hairpin resolvases. The 19 unique telomeres display initial rates of reaction in vitro that vary by over 160 -fold (20). The hairpin telomeres have an invariant box 3 that is recognized by the large proteolytic $\operatorname{Res} T$ fragment $\left(\operatorname{Res} T_{164-449)}\right)$ that carries the active site. The hairpin telomeres have been divided into three groups (Type 1,2 and 3) based upon the position or absence of box 1 sequences, which are bound by the $\mathrm{N}$-terminal domain $\left(\operatorname{Res} \mathrm{T}_{1-163}\right)$, which is delivered to the box 1 location by specific binding of ResT $T_{164-449}$ to box 3 (18). 
Type 2 telomeres have their box 1 offset by three base pairs in the direction of the axis of symmetry in a replicated telomere relative to Type 1 telomeres. Type 1 and Type 2 telomeres are both processed efficiently. Type 3 telomeres are lacking a box 1 and in general are processed much less efficiently than Type 1 and Type 2 telomeres. Three of the Type 3 telomeres were surprisingly, completely unreactive in vitro. The in vivo activity of the three in vitro unreactive telomeres was confirmed, pointing to a fundamental difference in reaction conditions in the living organism. This might be the presence of stimulatory proteins or a difference in DNA topology, such as positive supercoiling, that potentiates the reactivity of these Type 3 telomeres. Interestingly, the Type 2 telomeres display differential reactivity by ResT from Lyme spirochetes versus relapsing fever spirochetes (17). Through the use of chimeric and mutant ResT proteins the determinant conferring permissive substrate usage was localized to a single proline residue in the catalytic region that was absent in the relapsing fever enzymes but present in ResT from Lyme spirochetes (7).

\section{Reaction reversal and genome plasticity}

Another unusual feature in the genus Borrelia is the ongoing genome plasticity in the telomeric and subtelomeric regions of the linear chromosome and plasmids $(44,46-$ 49). Extensions of linear plasmid sequences can be found on the ends of some chromosomes and other linear plasmids (Fig. 5). In addition, the subterminal regions of many linear plasmids are a patchwork of repeated sequences found on other linear plasmids. Sequencing of recombination breakpoints indicates that these genome rearrangements result from non-homologous recombination events, however, no 
molecular mechanism for their generation was proposed $(44,46-48)$. A hint as to a possible molecular mechanism comes from the demonstration that telomere resolution by ResT is a reversible reaction (15). In the reverse reaction, referred to as telomere fusion, two hairpin telomeres on different DNA molecules are covalently linked to generate a replicated telomere structure (reversal of the reaction depicted in Fig.2). However, completely unrelated DNA molecules can be fused, generating chimeric plasmids or chromosomes with plasmid extensions. In the overwhelming majority of cases, these replicon fusions would be quickly resolved by the forward reaction, separating the fused replicons. However, at a very low frequency, the appearance of a mutation in the fused telomere would preclude resolution, resulting in a stabilized telomere fusion. Subsequent deletions would be expected to occur to remove one of the two sets of replication/maintenance functions on the replicon consisting of two fused plasmids. Such deletions could be quite large, removing large chunks of plasmid DNA and the stabilized fusions would be frozen in time and available to participate in subsequent telomere fusion and recombination events in the future. This type of processive mechanism can explain the patchwork of repeated sequences found in the subtelomeric regions of most of the linear Borrelia plasmids and the plasmid extensions found on some B. burgdorferi chromosomes (Fig. 5). Once established, the patchwork of repeats would also be available for homologous recombination reactions driven by the RecA pathway or perhaps by the strand exchange activity of ResT (23) discussed in a later section. For further discussion see $(1,2,15,23)$. 


\section{Domain diversity in hairpin telomere resolvases}

As noted above and in Fig. 3, hairpin telomere resolvases all share a catalytic core domain with an active site similar to that of type IB topoisomerases and tyrosine recombinases. However, these enzymes appear to have a multi-domain structure in which $\mathrm{N}$ - and C-terminal domain elaborations surrounding a central catalytic domain can be unrelated to each other in the different resolvases and likely have divergent functions (Fig. 6). For ResT, partial chymotrypsin cleavage, domain boundary mapping by mass spectroscopy and independent expression of the identified domains has uncovered twoproteolytic domains: an $\mathrm{N}$-terminal domain $\left(\operatorname{Res}_{1-163}\right)$ and a C-terminal domain, (Res $\left.T_{164-449}\right)$ (18). $R e s T_{1-163}$ terminates just after the hairpin-binding module that corresponds to the long linker $\alpha$-helix that connects the $\mathrm{N}$-terminal and catalytic domains of TelA and TelK $(12,18,25,26)$. In ResT, this domain possesses non-specific DNA binding activity apart from the hairpin-binding module. In the context of the full-length resolvase $\operatorname{Res}_{1-163}$ binds the box 1 sequences found near the centre of the $r T e l$ substrate (see Fig. 4). ResT $164-449$ encompasses the tyrosine recombinase-like catalytic domain (164-340) as well as an extension (341-449) that is likely an independently folded domain (18). The determinants of telomere recognition, mediated by the distally located box 3-5 sequences of the $r T e l$, are found in Res $T_{164-449}(18)$. The TelK hairpin resolvase possesses an extension of the C-terminal domain (the stirrup) that is required for telomere resolution (25). The stirrup domain makes site-specific contacts with distal portions of the $r T e l$, stabilizing a large distributed bend made in the $r T e l$ that contributes to hairpin formation (25). The stirrup is completely absent in the TelA resolvase (26). TelA, instead, possesses a short C-terminal extension that contributes to dimerization 
contacts (see Fig. 6). TelK and TelA have a shared $\mathrm{N}$-terminal core subdomain ( $\mathrm{N}$-core) that forms the linker and top of a C-clamp shaped embrace of the substrate DNA. TelK contains a large insertion in the $\mathrm{N}$-core domain, called the muzzle, which makes extensive interactions required for dimerization and is essential for telomere resolution (25). TelA has an additional $\mathrm{N}$-terminal subdomain in addition to the $\mathrm{N}$-core that is dispensable for telomere resolution (26).

An intriguing aspect of the domain arrangement of ResT is the observation that the determinants of telomere recognition harboured in the $\operatorname{Res}_{164-449}$ domain are partially masked in the full-length resolvase, either by steric hinderence from $\operatorname{Res} T_{1-163}$ or by a conformational change in the full-length enzyme (18). The conserved Box 3 sequence (TTAGTATA) that ResT recognizes in the $r T e l$ is present in thousands of copies in the genome; the autoinhibition of telomere binding may act to down regulate ResT binding and activity until a genuine cross-axis complex can be formed at bona fide replicated telomere substrates (18).

\section{Requirement for the hairpin-binding module of ResT for DNA cleavage}

In addition to the tyrosine recombinase-like active site, ResT employs a distinct part of the protein located at the end of the N-terminal domain (positions 139-159) called the hairpin-binding module $(50,51)$, to license DNA cleavage by the catalytic domain. A hairpin binding module is not present in TelK or TelA. A similarity of this part of ResT to the hairpin-binding module of IS4 transposases was noted and investigated (12). The hairpin-binding module in these transposases is composed of a hydrophobic pocket and an $\alpha$-helix with charged residues. The hairpin-binding module stabilizes the transient 
hairpin intermediate that arises during transposon excision. The hydrophobic pocket stacks on a flipped-out base that changes the normal trajectory of the cleaved DNA strand so that it can form the hairpin intermediate. A series of electrostatic interactions between the charged residues on the a-helix and the DNA backbone further stabilizes the hairpin intermediate $(50,51)$. Mutants in the transposase hairpin-binding module have defects in hairpin formation (52-55). Mutation of the analogous positions in ResT resulted in variants defective in telomere resolution, most notably in the analogue of the hydrophobic pocket, and cold-sensitive variants when charged residues were mutated. These mutants were defective for DNA cleavage but were readily and specifically rescued by introduction of heteroduplex DNA at the central two base pairs in the centre of the $r T e l(12)$. The rescued reactions on heteroduplexed $r T e / s$ proceeded to hairpin telomere formation rather than just rescuing the DNA cleavage reaction. This implies that the DNA distortions in the $r T e /$ induced by the ResT hairpin-binding module that are mimicked by the heteroduplex, license DNA cleavage but also promote subsequent hairpin formation (12). The DNA distortion induced by the hairpin-binding module is distinct from that which promotes cross-axis formation since the central nick that promotes cross-axis complex formation does not rescue hairpin-binding module mutants (12). The precise nature of the distortion and the role played by individual sidechains within the hairpin-binding module remain to be elucidated. In contrast to the requirement for a ResT region involved in hairpin formation, for DNA cleavage, TelA mutations in the $\mathrm{N}$-core domain in key linker helix residues that appear to direct strand refolding (Y201A and R205A) are reported as cleavage competent but defective for hairpin formation (26). However, the observation that these mutants require days or weeks of incubation with 
suicide half-site substrates for cleavage, point to a possible additional role for these residues in early interactions with the substrate DNA to allow cleavage.

\section{Hairpin formation/strand foldback}

In the model of hairpin telomere formation based on the TelK-cleaved $r T e /$ complex it was inferred that torsion stored in the distributed out-of-plane bend in the DNA site drives dimer dissolution and spontaneous strand foldback after product release (25). This model was motivated by considerations of steric clash between hairpins that might form in the context of the extensively interlocked dimer (Fig. 7) and by the lack of contacts involving TelK and the DNA between the scissile phosphates. This model was also supported by the observation that deletion of the stirrup (C-terminal) subdomain, important for forming and stabilizing the substrate DNA bend, produced a TelK variant that was cleavage competent but defective in hairpin formation (25).

In contrast, biochemical studies on ResT demonstrated that hairpin telomere formation must occur in the context of the dimer of ResT bound to the $r T e l$, implying an active role for the telomere resolvase in the strand foldback required for hairpin telomere formation (22). These experiments made use of bead immobilized telomere resolution assays in which the $r T e /$ substrate was linked via one strand to paramagnetic beads by a 3'-biotin moiety. In this assay system, telomere resolution produces hairpin telomeres of distinct size: one remains attached to the bead and one hairpin is released into the supernatant fluid. Bead and supernatant fractions are separated by bead pull down followed by gel analysis of the fractions. This experimental set-up allowed for investigation of issues of product release. Fortuitous and deliberately engineered $r \mathrm{Tel}$ 
substrates that showed a large bias in the speed and efficiency of hairpin telomere formation on the two halves of the substrate in free reactions were assayed in bead immobilized form. It was discovered that hairpin telomere product was not released into the supernatant until both hairpins had been formed (22). These results indicated an active role for ResT in hairpin formation. The observed hairpin product release for ResT is in contrast to the binding of the hairpin telomeres within a dimeric product complex observed for the TelA hairpin resolvase $(8,26)$.

The TelA-hairpin telomere product complexes as well as structures of TelA with suicide substrates that prevent hairpin formation argued for an active enzyme-mediated strand foldback mechanism of telomere formation (26). Structures of the product complex feature a dimer of TelA with the hairpin telomeres folded into an extremely compact conformation; steric clash between the hairpins is avoided by a difference in the helix trajectory in the two hairpins, breaking the 2-fold symmetry of the complex (Fig. 7). TelA makes extensive stabilizing interactions with the hairpin turnaround, including with residues in the linker $\alpha$-helix that comprises the end of the $\mathrm{N}$-core domain, a region of TelA that occupies the same relative position in TelA as the hairpin-binding module of ResT. Structures with suicide substrates that prevent hairpin formation identified a 'refolding intermediate' in which the refolding DNA strands assume an open conformation featuring extrahelical bases in a linearly stacked arrangement. The crystal structure also reveals Hoogstein base-pairing across the symmetry axis and interactions with key hairpin-interacting residues that stack an extrahelical base and otherwise constrain the possible trajectory of the refolding strands (26). 
The overall structure of the $\mathrm{N}$-terminal core and catalytic domain of TelA that was present in the structures was found be very similar to that of TelK. Moreover, the feature of the dimer complex being offset by a 'jog' in the area that corresponds to the helix between the scissile phosphates in the TelK-cleaved substrate complex was also present (see Fig. 7). The $\alpha$-helical linker of the $\mathrm{N}$-core domain blocks the helical path that substrate DNA would have to assume to fit into the TelA dimer complex. This argues that the substrate DNA would likely have to be severely distorted or unwound during initial interactions with the resolvase. Such a distortion, believed to be made by the hairpin-binding module in ResT, allows productive engagement of the catalytic domains for DNA cleavage, blocks resealing of parental strands and promotes subsequent hairpin formation $(12,14)$.

\section{Holliday junction formation by ResT}

A further dramatic similarity of ResT to the tyrosine recombinases was uncovered under conditions known to inhibit telomere resolution, including low incubation temperature or negative DNA supercoiling in the substrate. Under these conditions ResT can synapse together rTels and catalyze the formation of a Holliday junction (21). The simplest tyrosine recombinases are typified by Flp and Cre. They produce inversion or deletion recombinants from reaction sites composed of simple inverted repeat sequences that direct recombinase binding, coupled with sequence asymmetry in the central region of the reaction site between the scissile phosphates. The central sequence asymmetry directs strand exchange between sites synapsed in an antiparallel orientation and directs a preferred order of strand cleavage and exchange that 
promotes the formation of recombinant products (56-60). Replicated telomere substrates for ResT lack such directional cues and consequently the HJs formed from symmetric $r T e / s$ lacked directionality (21). Artificially asymmetrizing $r T e /$ sites so that they progressively appeared more like recombination sites resulted in progressive inhibition of telomere resolution with a corresponding increase in directional HJ formation. Since replicated telomeres in vivo will always be symmetric it is unlikely that ResT catalyzes site-specific recombination in vivo. It is simpler to posit that this activity reflects the evolutionary origin of telomere resolvases as ancestral phage integrases or cellular plasmid/chromosome dimer resolvases (see $(2,21))$. Consistent with this idea is the close structural similarity of the catalytic domain of TelK and TelA to that of $\lambda$ integrase $(25,26)$. This contention is also supported by experiments demonstrating a one-step linearization of the E. coli chromosome without deleterious effects on cell growth (61). The chromosome was linearized by introduction of the phage N15 replicated telomere near the $E$. coli replication terminus, coupled with the expression of the N15 telomere resolvase, TelN.

The discovery of ResT's ability to synapse rTe/s and to catalyze HJ formation raised the interesting possibility that telomere resolution might occur in the context of a synapse of $r T e / s$ and that $\mathrm{HJ}$ formation is a possible side-reaction that occurs when hairpin formation is inhibited in some fashion. This possibility had some attractive features since it offered a mechanism for linking telomere resolution to cell division, similar to the dynamics of chromosome dimer resolution catalyzed by Xer/dif system (34, $62,63)$. Synapsis-dependent models of telomere resolution were tested and definitively 
ruled out by assays with replicated telomeres immobilized at sub-saturating conditions on streptavidin-coated paramagnetic beads (22).

\section{A possible role for ResT in homologous recombination of linear replicons}

Recently, an unexpected activity of ResT was uncovered: a vigorous single-strand DNA annealing activity (SSA) and the ability to promote DNA strand exchange between single-stranded donor DNAs and homologous partial duplex target DNAs (23). Interestingly, the ResT N-terminal domain, $\operatorname{Res}_{1-163}$ was found to promote SSA and strand exchange on its own (23). The properties of these reactions promoted by ResT are consistent with a mode of action similar to single-strand annealing proteins (SSAPs) typified by phage $\lambda$ Beta of the $\lambda$ Red recombination system and of Rad52 the eukaryotic SSAP that participates in RecA/Rad51-dependent and -independent homologous recombination reactions (64). ResT was found to be able to support SSA between complementary or partially complementary strands of up to $106 \mathrm{nt}$ in length and to promote strand exchange through up to $63 \mathrm{bp}$ of duplex DNA. Strand exchange was found to be sensitive to heterology between the ssDNA donor and the exchanging segment of the partial duplex target, giving rise to double-flap complexes that have strand exchanged beyond the heterology, but that have stalled shortly thereafter. Such events, if they occur in vivo, would generate short-patch recombinants. Such short patch recombinants typify the gene conversion events found in switching at the $B$. burgdorferi $V / s E$ locus to promote antigenic variation (65). The mechanism of switching at $v / s E$ remains uncharacterized and the only host factor known to participate in the reaction is the RuvAB branch migrase $(66,67)$. It is tempting to speculate that ResT might play a 
role in recombination at $v / s E$. Alternatively, ResT could play a possible role in homologous recombination reactions or perhaps for some, as yet, unappreciated role in hairpin telomere biology. Evidence has been presented that $B$. burgdorferi telomeres are recombinogenic $(2,10)$ and the possibility exists that the strand exchange activity that resides in $\operatorname{Res}_{1-163}$ may play a role in these recombination events.

\section{In vivo effects of a conditional resT mutant}

Our current understanding of the telomere resolution process began with the demonstration that the process occurred in vivo to generate the hairpin telomeres in $B$. burgdorferi (10). Thereafter, a large body of data has been generated through biochemical experiments with purified proteins and DNA substrates and by structural studies. However, at least for ResT, there is not a complete congruence of in vivo and in vitro reaction properties. In particular, some telomere substrates that are active in vivo show no detectable activity in vitro (20). In addition, Type 2 telomeres cannot be processed in vitro by relapsing fever telomere resolvases, but are efficiently utilized in vivo (17). It is reasonable to postulate that the reaction in vivo is stimulated by other proteins or by substrate topology, such as positive supercoiling (16). Moreover, we know little about whether telomere resolvases interact with other proteins within the cell and how they may be involved with regulation of other cellular processes such as replication initiation, elongation and cell division.

As one might expect, the res $T$ gene on cp26 of $B$. burgdorferi cannot be disrupted (68) unless a complementing res $T$ gene has been added to the cell (17). To further study the in vivo activities of this essential gene, a strain with conditional expression of 
res $T$ was constructed (69). The res $T$ gene was cloned under the control of an IPTGinducible expression system and the endogenous gene was disrupted. The protein was found to be expressed at about 15,000 monomers per cell, which is on the high side for a replication protein, suggesting that it may have other functions. Removal of IPTG from the culture allowed determination of the half-life of ResT to be 16 hours. ResT depletion resulted in growth arrest of the spirochetes at 48 hours. Filamentous forms did not appear, as might be expected if telomere resolution were linked to cell division. DNA replication did not continue after growth arrest, suggesting that ResT might interact directly or indirectly with factors controlling the initiation or elongation of DNA synthesis. Simple dimeric replication intermediates of linear plasmids were only visible at 24-48 hours after IPTG washout after which time they morphed into higher molecular weight forms. These more complex molecules may result from additional replication initiation events or stalled replication. Further studies will be required to elucidate the in vivo activities and interactions of ResT.

\section{Acknowledgements}

Thw authors would like to thank Phoebe Rice for structural comparisons to define domain boundaries and for generating figures $6 \mathrm{~B}$ and $\mathrm{C}$ and 7 . Work in the authors' laboratories was supported by grant MOP 53086 from the Canadian Institutes of Health Research to G.C.(http://www.cihr-irsc.gc.ca/e/193.html) by grant MOP 79344 from the Canadian Institutes of Health Research to K.K., by grant RGPIN 326797-2011 from the Natural Sciences \& Engineering Research Council of Canada to K.K. (http://www.nserc-

crsng.gc.ca/index eng.asp) and by grant 2570 from the Saskatchewan Health Research 
Fund to K.K. (http://shrf.ca/).G.C. holds a Canada Research Chair in the Molecular Biology of Lyme Borreliosis (http://www.chairs-chaires.gc.ca/home-accueil-eng.aspx) and a Scientist Award from Alberta Innovates - Health Solutions (http://www.ahfmr.ab.ca/).

\section{Figure Legends}

Fig. 1. Hairpin telomere resolution as a solution to the end-replication problem.

Replication of a linear molecule results in the formation of dimer junctions or replicated telomeres (L'L, RR') that are processed by telomere resolution, a unique type of DNA breakage and reunion reaction. Telomere resolution results in the formation of hairpin telomeres at the ends of the linear DNA molecule and separates the dimer replication intermediate into monomeric products of DNA replication. See text for further details. This figure is adapted from (6) and reprinted from (1).

Fig. 2. ResT promotes telomere resolution via a two-step transesterification. 1) The telomere resolvase, ResT, binds to a replicated telomere (rTel) substrate, which corresponds to the dimer junction L'L or RR' shown in Fig. 1 (11). The black dots denote the scissile phosphates and the vertical bar in the center of $r T e l$ the axis of 180-degree rotational symmetry. 2) Positive supercoiling is believed to facilitate the cooperative formation of a cross-axis complex where communication between ResT protomers bound on both sides of the symmetry axis occurs $(14,16) .3)$ The action of the "hairpin binding module", a region with sequence similarity to a motif found in cut-and-paste 
transposases, induces a DNA distortion that facilitates 4) DNA cleavage, the first transesterification event (12). 5) A conformational change then occurs to juxtapose the free 5'-OH groups to the 3-phosphotyrosyl enzyme intermediates on the opposite strands. 6) Nucleophilic attack of the phosphotyrosyl linkage by the 5'-OH groups, the second transesterification step, results in phosphodiester bond formation to stabilize the hairpins generated during step 5. This figure and legend is adapted from (43) and (21), and reprinted from (2).

Fig. 3. Sequence alignment of the domains carrying the active site residues of purified telomere resolvases. An alignment is shown for the telomere resolvases from the phages N15, TelN (3), $\varphi \mathrm{KO}$, TelK (4), PY54, TelY (5) and the bacterial resolvases from Agrobacterium tumefaciens, TelA (8), the Lyme spirochete Borrelia burgdorferi, ResTBb (6) and the relapsing fever species Borrelia hermsii, ResTBh (7). Several other purified hairpin resolvases from othe Borrelia species are not included in the lineup. The catalytic residues are indicated by asterisks and the active site tyrosine by a red asterisk. The double colon indicates the position of the proline in $B$. burgdorferi ResT that confers permissiveness for Type 2 telomeres (7). The corresponding active site residues for the tyrosine recombinase family are indicated above and below the alignment in red and those for type IB topoisomerases in blue.

Fig. 4. B. burgdorferi telomere sequence alignment. Telomere sequences are arranged in descending order, according to the initial rate of telomere resolution. The initial rate, expressed in $\mathrm{fmol} / \mathrm{min}$ is shown in the right hand column and telomere sequences are 
aligned with the hairpins (or symmetry axis in the replicated telomeres) at the left end. The telomeres shown are half of the actual replicated telomere substrates used in the telomere resolution reactions. The colored boxes labeled 1 and 3 refer to previously identified regions of sequence homology (11), with some modifications. The original box 1 sequence, TATAAT is indicated by a light blue box, while the newly identified box 1 sequence, TATTAT is shown in dark blue. The homology box 3 region has been expanded from the five nucleotide sequence TAGTA to the eight nucleotide sequence TTAGTATA. The telomere sequences of Ip17L, Ip17R, Ip21R, Ip28-1R, Ip56R ChromL and ChromR have been reported previously $(45,47,48,70-72)$. Reprinted from (20).

Fig. 5. Telomere exchanges believed to be mediated by ResT promoted telomere fusions. The proposed mechanism for telomere exchange between linear plasmids and the right end of the $B$. burgdorferi chromosome is a two step process. The first is a telomere fusion event and the second is a deletion or other type of mutation to inactivate or remove the newly fused telomere and prevent its resolution and to remove competeing replication maintenance functions. The telomere fusion event is promoted by reversal of the ResT reaction such that two hairpin telomeres from different molecules are fused to generate a single DNA molecule carrying a replicated telomere. A deletion removing the telomere resolution site might be specifically targeted to the fused telomere by incomplete joining in the reverse reaction, to leave a ResT molecule covalently linked at a nick in the telomere; such covalent protein-DNA complexes are known to be foci for the formation of deletions and other chromosomal aberrations (73). Alternatively, a deletion could be derived from palindrome instability induced by passage 
of a replication fork through the inverted repeat of the fused telomere $(74,75)$. $B$. burgdorferi chromosome extensions that may have arisen by ResT-mediated telomere fusions followed by deletion formation (see $(1,2,15)$ ). The B31 chromosome appears to have arisen from fusion of an Ip28-1 plasmid with the N40 chromosome. Subsequently, a single fusion of the B31 chromosome with Ip21, followed by sequence deletion would have generated the 297 chromosome. Similarly, two rounds of fusion/deletion of the B31 chromosome, first with an Ip28-1 and subsequently with Ip28-5, would have generated the JD1 chromosome. The sequence relatedness of the chromosomes and plasmids shown were reported by $(44,46-48)$. This figure is slightly modified from (46).

Fig. 6. The domain structure of hairpin telomere resolvases. A) The domain composition of the telomere resolvases from Klebsiella phage $\phi K O 2$ (TelK), Agrobacterium (TelA) and Borrelia species (ResT) are shown (see also text). All telomere resolvases have a central catalytic domain with active site residues similar those of tyrosine recombinases and type IB topoisomerses (see Fig 3 and text). In addition, hairpin resolvases carry divergent $\mathrm{N}$-terminal domains; they may also carry a divergent $\mathrm{C}$-terminal region or it may be absent as for TelA. Domains represented by the same shape and colour scheme are structurally related. Domains delimited by brackets represent protein sequences dispensable for telomere resolution in vitro $(8,25)$. Also shown for each hairpin telomere resolvase is the tyrosine nucleophile $(\mathrm{Y})$. The numbers above the graphics refer to the amino acid numbers of the proteins. Precise domain boundaries have been adjusted from those previously reported based upon structural alignments. B and C) A structural view of the domain organization of TelK and TelA, respectively. The 
domains are represented by the same colour scheme used in A) on one monomer in each dimer. The $\mathrm{N}$-core domain, represented in blue, is composed of a helical bundle that forms the top of the C-clamp embrace the resolvases make with the substrate DNA and the long linker $\alpha$-helix that connects the top and bottom of the C-clamp. TelK has a large insertion in the $\mathrm{N}$-core domain called the muzzle; this is represented in yellow. The shared catalytic domain is represented in red. TelK has an additional C-terminal domain essential for telomere resolution called the stirrup; this domain is represented in grey. Beyond the shared catalytic domain TelA lacks an additional domain like the stirrup but instead has a short segment that contributes to dimerization contacts, this short Cterminal extension is represented in green. The arrows with residue numbers indicate the position in the structure of the first and last resolvable residues present in the structures. The structures presented were generated with The PyMOL Molecular Graphics System, Version 1.7 Schrödinger, LLC. (http://www.pymol.org/) using PDB ID $2 \mathrm{v} 6 \mathrm{e}$ for TelK (25) and $4 \mathrm{e} 0 \mathrm{~g}$ for TelA (26).

Fig. 7. TelK and TelA-DNA complexes showing displacement of the helical axes at the dimer interface by 7.5 and $>10$ Angstroms, respectively. The structures presented were generated with The PyMOL Molecular Graphics System, Version 1.7 Schrödinger, LLC. (http://www.pymol.org/) using PDB ID 2v6e for TelK (25) and 4e0g for TelA (26).

\section{References}

1. Chaconas. 2005. Hairpin telomeres and genome plasticity in Borrelia: all mixed up in the end. Molecular Microbiology 58:625-635. 
2. Chaconas G, Kobryn K. 2010. Structure, function, and evolution of linear replicons in Borrelia. Annu Rev Microbiol 64:185-202.

3. Deneke J, Ziegelin G, Lurz R, Lanka E. 2000. The protelomerase of temperate Escherichia coli phage N15 has cleaving-joining activity. Proc Natl Acad Sci U S A $97: 7721-7726$.

4. Huang WM, Joss L, Hsieh T, Casjens S. 2004. Protelomerase uses a topoisomerase IB/Y-recombinase type mechanism to generate DNA hairpin ends. Journal of molecular biology 337:77-92.

5. Hertwig S, Klein I, Lurz R, Lanka E, Appel B. 2003. PY54, a linear plasmid prophage of Yersinia enterocolitica with covalently closed ends. Mol Microbiol 48:989-1003.

6. Kobryn K, Chaconas G. 2002. ResT, a telomere resolvase encoded by the Lyme disease spirochete. Mol Cell 9:195-201.

7. Moriarty TJ, Chaconas G. 2009. Identification of the determinant conferring permissive substrate usage in the telomere resolvase, ResT. The Journal of biological chemistry 284:23293-23301.

8. Huang WM, DaGloria J, Fox H, Ruan Q, Tillou J, Shi K, Aihara H, Aron J, Casjens S. 2012. Linear chromosome-generating system of Agrobacterium 
tumefaciens C58: protelomerase generates and protects hairpin ends. The Journal of biological chemistry 287:25551-25563.

9. Ramirez-Bahena MH, Vial L, Lassalle F, Diel B, Chapulliot D, Daubin V, Nesme X, Muller D. 2014. Single acquisition of protelomerase gave rise to speciation of a large and diverse clade within the Agrobacterium/Rhizobium supercluster characterized by the presence of a linear chromid. Mol Phylogenet Evol. 73: 202-207.

10. Chaconas G, Stewart PE, Tilly K, Bono JL, Rosa P. 2001. Telomere resolution in the Lyme disease spirochete. EMBO J 20:3229-3237.

11. Tourand Y, Kobryn K, Chaconas G. 2003. Sequence-specific recognition but position-dependent cleavage of two distinct telomeres by the Borrelia burgdorferi telomere resolvase, ResT. Mol Microbiol 48:901-911.

12. Bankhead T, Chaconas G. 2004. Mixing active site components: A recipe for the unique enzymatic activity of a telomere resolvase. Proc. Natl. Acad. Sci. USA 101:13768-13773.

13. Deneke J, Burgin AB, Wilson SL, Chaconas G. 2004. Catalytic residues of the telomere resolvase ResT: a pattern similar to, but distinct from tyrosine recombinases and type IB topoisomerases. J. Biol. Chem. 279:53699-53706. 
14. Kobryn K, Burgin AB, Chaconas G. 2005. Uncoupling the chemical steps of telomere resolution by ResT. The Journal of biological chemistry 280:2678826795.

15. Kobryn K, Chaconas G. 2005. Fusion of hairpin telomeres by the B. burgdorferi telomere resolvase ResT: Implications for shaping a genome in flux. Mol Cell 17:783-791.

16. Bankhead T, Kobryn K, Chaconas G. 2006. Unexpected twist: harnessing the energy in positive supercoils to control telomere resolution. Mol Microbiol 62:895905.

17. Tourand Y, Bankhead T, Wilson SL, Putteet-Driver AD, Barbour AG, Byram R, Rosa PA, Chaconas G. 2006. Differential telomere processing by Borrelia telomere resolvases in vitro but not in vivo. J Bacteriol 188:7378-7386.

18. Tourand Y, Lee L, Chaconas G. 2007. Telomere resolution by Borrelia burgdorferi ResT through the collaborative efforts of tethered DNA binding domains. Mol Microbiol 64:580-590.

19. Lefas G, Chaconas G. 2009. High-throughput screening identifies three inhibitor classes of the telomere resolvase from the Lyme disease spirochete. Antimicrobial Agents and Chemotherapy 53:4441-4449. 
20. Tourand Y, Deneke J, Moriarty TJ, Chaconas G. 2009. Characterization and in vitro reaction properties of 19 unique hairpin telomeres from the linear plasmids of the Lyme disease spirochete. The Journal of biological chemistry 284:7264-7272.

21. Kobryn K, Briffotaux J, Karpov V. 2009. Holliday junction formation by the Borrelia burgdorferi telomere resolvase, ResT: implications for the origin of genome linearity. Mol Microbiol 71:1117-1130.

22. Briffotaux J, Kobryn K. 2010. Preventing broken borrelia telomeres: Rest couples dual hairpin telomere formation to product release. The Journal of biological chemistry. 285: 41010-41018.

23. Mir T, Huang SH, Kobryn K. 2013. The telomere resolvase of the Lyme disease spirochete, Borrelia burgdorferi, promotes DNA single-strand annealing and strand exchange. Nucleic acids research 41:10438-10448.

24. Deneke J, Ziegelin G, Lurz R, Lanka E. 2002. Phage N15 telomere resolution: Target requirements for recognition and processing by the protelomerase. The Journal of biological chemistry 277:10410-10419.

25. Aihara H, Huang WM, Ellenberger T. 2007. An interlocked dimer of the protelomerase TelK distorts DNA structure for the formation of hairpin telomeres. Mol Cell 27:901-913. 
26. Shi K, Huang WM, Aihara H. 2013. An enzyme-catalyzed multistep DNA refolding mechanism in hairpin telomere formation. PLoS Biol 11:e1001472.

27. Shuman S. 1998. Vaccinia virus DNA topoisomerase: a model eukaryotic type IB enzyme. Biochim Biophys Acta 1400:321-337.

28. Van Duyne GD. 2002. A structural view of tyrosine recombinase site-specific recombination, p. 93-117. In Craig NL, Craigie, R., Gellert, M. and Lambowitz, A.M. (ed.), Mobile DNA II. ASM Press, Washington, D.C.

29. Grindley ND, Whiteson KL, Rice PA. 2006. Mechanisms of site-specific recombination. Annu Rev Biochem 75:567-605.

30. Lee J, Tonozuka T, Jayaram M. 1997. Mechanism of active site exclusion in a site-specific recombinase: role of the DNA substrate in conferring half-of-the-sites activity. Genes Dev 11:3061-3071.

31. Voziyanov Y, Pathania S, Jayaram M. 1999. A general model for site-specific recombination by the integrase family recombinases. Nucleic acids research 27:930-941.

32. Conway AB, Chen Y, Rice PA. 2003. Structural plasticity of the Flp-Holliday junction complex. Journal of molecular biology 326:425-434. 
33. Burgin AB, Jr., Nash HA. 1995. Suicide substrates reveal properties of the homology-dependent steps during integrative recombination of bacteriophage lambda. Curr Biol 5:1312-1321.

34. Barre FX, Aroyo M, Colloms SD, Helfrich A, Cornet F, Sherratt DJ. 2000. FtsK functions in the processing of a Holliday junction intermediate during bacterial chromosome segregation. Genes Dev 14:2976-2988.

35. Rybchin VN, Svarchevsky AN. 1999. The plasmid prophage N15: a linear DNA with covalently closed ends. Mol Microbiol 33:895-903.

36. Krogh BO, Shuman S. 2000. Catalytic mechanism of DNA topoisomerase IB. Mol Cell 5:1035-1041.

37. Krogh BO, Shuman S. 2002. Proton relay mechanism of general acid catalysis by DNA topoisomerase IB. The Journal of biological chemistry 277:5711-5714.

38. Burgin AB. 2001. Synthesis and use of DNA containing a 5'-bridging phosphorothioate as a suicide substrate for type I DNA topoisomerases. Methods Mol Biol 95:119-128.

39. Van Duyne GD. 2001. A structural view of cre-loxp site-specific recombination. Annu Rev Biophys Biomol Struct 30:87-104. 
40. Chen Y, Rice PA. 2003. The role of the conserved Trp330 in Flp-mediated recombination. Functional and structural analysis. The Journal of biological chemistry 278:24800-24807.

41. Whiteson KL, Chen Y, Chopra N, Raymond AC, Rice PA. 2007. Identification of a potential general acid/base in the reversible phosphoryl transfer reactions catalyzed by tyrosine recombinases: Flp H305. Chemistry \& biology 14:121-129.

42. Davies DR, Mushtaq A, Interthal H, Champoux JJ, Hol WG. 2006. The structure of the transition state of the heterodimeric topoisomerase I of Leishmania donovani as a vanadate complex with nicked DNA. Journal of molecular biology 357:1202-1210.

43. Kobryn K. 2007. The linear hairpin replicons of Borrelia burgdorferi, p. 117-140. In Meinhardt F, Klassen R (ed.), Microbial Linear Plasmids. Springer, Berlin Heidelberg.

44. Casjens S, Palmer N, van Vugt R, Huang WH, Stevenson B, Rosa P, Lathigra R, Sutton G, Peterson J, Dodson RJ, Haft D, Hickey E, Gwinn M, White O, Fraser CM. 2000. A bacterial genome in flux: the twelve linear and nine circular extrachromosomal DNAs in an infectious isolate of the Lyme disease spirochete Borrelia burgdorferi. Mol Microbiol 35:490-516. 
45. Fraser CM, Casjens S, Huang WM, Sutton GG, Clayton R, Lathigra R, White O, Ketchum KA, Dodson R, Hickey EK, Gwinn M, Dougherty B, Tomb JF, Fleischmann RD, Richardson D, Peterson J, Kerlavage AR, Quackenbush J, Salzberg S, Hanson M, van Vugt R, Palmer N, Adams MD, Gocayne J, Weidman J, Utterback T, Watthey L, McDonald L, Artiach P, Bowman C, Garland S, Fujii C, Cotton MD, Horst K, Roberts K, Hatch B, Smith HO, Venter JC. 1997. Genomic sequence of a Lyme disease spirochaete, Borrelia burgdorferi. Nature 390:580-586.

46. Casjens SR, Mongodin EF, Qiu WG, Luft BJ, Schutzer SE, Gilcrease EB, Huang WM, Vujadinovic M, Aron JK, Vargas LC, Freeman S, Radune D, Weidman JF, Dimitrov GI, Khouri HM, Sosa JE, Halpin RA, Dunn JJ, Fraser CM. 2012. Genome stability of Lyme disease spirochetes: comparative genomics of Borrelia burgdorferi plasmids. PloS one 7:e33280.

47. Huang WM, Robertson M, Aron J, Casjens S. 2004. Telomere exchange between linear replicons of Borrelia burgdorferi. J Bacteriol 186:4134-4141.

48. Casjens S, Murphy M, DeLange M, Sampson L, van Vugt R, Huang WM. 1997. Telomeres of the linear chromosomes of Lyme disease spirochaetes: nucleotide sequence and possible exchange with linear plasmid telomeres. Mol Microbiol 26:581-596. 
49. Terekhova D, lyer R, Wormser GP, Schwartz I. 2006. Comparative genome hybridization reveals substantial variation among clinical isolates of Borrelia burgdorferi sensu stricto with different pathogenic properties. J Bacteriol 188:6124-6134.

50. Davies DR, Goryshin IY, Reznikoff WS, Rayment I. 2000. Three-dimensional structure of the Tn5 synaptic complex transposition intermediate. Science 289:7785.

51. Lovell S, Goryshin IY, Reznikoff WR, Rayment I. 2002. Two-metal active site binding of a Tn5 transposase synaptic complex. Nat Struct Biol 9:278-281.

52. Ason B, Reznikoff WS. 2002. Mutational analysis of the base flipping event found in Tn5 transposition. The Journal of biological chemistry 277:11284-11291.

53. Allingham JS, Wardle SJ, Haniford DB. 2001. Determinants for hairpin formation in Tn10 transposition. EMBO J 20:2931-2942.

54. Bischerour J, Chalmers R. 2007. Base-flipping dynamics in a DNA hairpin processing reaction. Nucleic acids research 35:2584-2595.

55. Bischerour J, Chalmers R. 2009. Base flipping in tn10 transposition: an active flip and capture mechanism. PloS one 4:e6201. 
56. Ghosh K, Lau CK, Gupta K, Van Duyne GD. 2005. Preferential synapsis of loxP sites drives ordered strand exchange in Cre-loxP site-specific recombination. Nature chemical biology 1:275-282.

57. Hoess RH, Wierzbicki A, Abremski K. 1986. The role of the loxP spacer region in $\mathrm{P} 1$ site-specific recombination. Nucleic acids research 14:2287-2300.

58. Lee L, Chu LC, Sadowski PD. 2003. Cre induces an asymmetric DNA bend in its target loxP site. The Journal of biological chemistry 278:23118-23129.

59. Lee L, Sadowski PD. 2003. Sequence of the loxP site determines the order of strand exchange by the Cre recombinase. Journal of molecular biology 326:397412.

60. Senecoff JF, Cox MM. 1986. Directionality in FLP protein-promoted site-specific recombination is mediated by DNA-DNA pairing. The Journal of biological chemistry 261:7380-7386.

61. Cui T, Moro-oka N, Ohsumi K, Kodama K, Ohshima T, Ogasawara N, Mori H, Wanner B, Niki H, Horiuchi T. 2007. Escherichia coli with a linear genome. EMBO Rep 8:181-187. 
62. Aussel L, Barre FX, Aroyo M, Stasiak A, Stasiak AZ, Sherratt D. 2002. FtsK Is a DNA motor protein that activates chromosome dimer resolution by switching the catalytic state of the XerC and XerD recombinases. Cell 108:195-205.

63. Bigot S, Saleh OA, Lesterlin C, Pages C, El Karoui M, Dennis C, Grigoriev M, Allemand JF, Barre FX, Cornet F. 2005. KOPS: DNA motifs that control E. coli chromosome segregation by orienting the FtsK translocase. Embo J 24:37703780.

64. Kuzminov A. 1999. Recombinational repair of DNA damage in Escherichia coli and bacteriophage lambda. Microbiol Mol Biol Rev 63:751-813.

65. Coutte L, Botkin DJ, Gao L, Norris SJ. 2009. Detailed analysis of sequence changes occurring during vlsE antigenic variation in the mouse model of Borrelia burgdorferi infection. PLoS Pathog 5:e1000293.

66. Dresser AR, Hardy P-O, Chaconas G. 2009. Investigation of the role of DNA replication, recombination and repair genes in antigenic switching at the vlsE locus in Borrelia burgdorferi: an essential role for the RuvAB branch migrase. PLoS Pathogens 5:e1000680.

67. Lin T, Gao L, Edmondson DG, Jacobs MB, Philipp MT, Norris SJ. 2009. Central role of the Holliday junction helicase RuvAB in vIsE recombination and infectivity of Borrelia burgdorferi. PLoS Pathogens 12:e1000679. 
68. Byram R, Stewart PE, Rosa P. 2004. The essential nature of the ubiquitous 26kilobase circular replicon of Borrelia burgdorferi. J Bacteriol 186:3561-3569.

69. Bandy NJ, Salman-Dilgimen A, Chaconas G. 2014. Construction and characterization of a B. burgdorferi strain with conditional expression of the essential telomere resolvase, ResT. manuscript submitted.

70. Hinnebusch J, Barbour AG. 1991. Linear plasmids of Borrelia burgdorferi have a telomeric structure and sequence similar to those of a eukaryotic virus. $\mathrm{J}$ Bacteriol 173:7233-7239.

71. Hinnebusch J, Bergstrom S, Barbour AG. 1990. Cloning and sequence analysis of linear plasmid telomeres of the bacterium Borrelia burgdorferi. Mol Microbiol 4:811-820.

72. Zhang JR, Hardham JM, Barbour AG, Norris SJ. 1997. Antigenic variation in Lyme disease borreliae by promiscuous recombination of VMP-like sequence cassettes. Cell 89:275-285.

73. Froelich-Ammon SJ, Osheroff N. 1995. Topoisomerase poisons: harnessing the dark side of enzyme mechanism. The Journal of biological chemistry 270:21429-21432. 
74. Pinder DJ, Blake CE, Lindsey JC, Leach DR. 1998. Replication strand preference for deletions associated with DNA palindromes. Mol Microbiol 28:719727.

75. Leach DR, Okely EA, Pinder DJ. 1997. Repair by recombination of DNA containing a palindromic sequence. Mol Microbiol 26:597-606. 

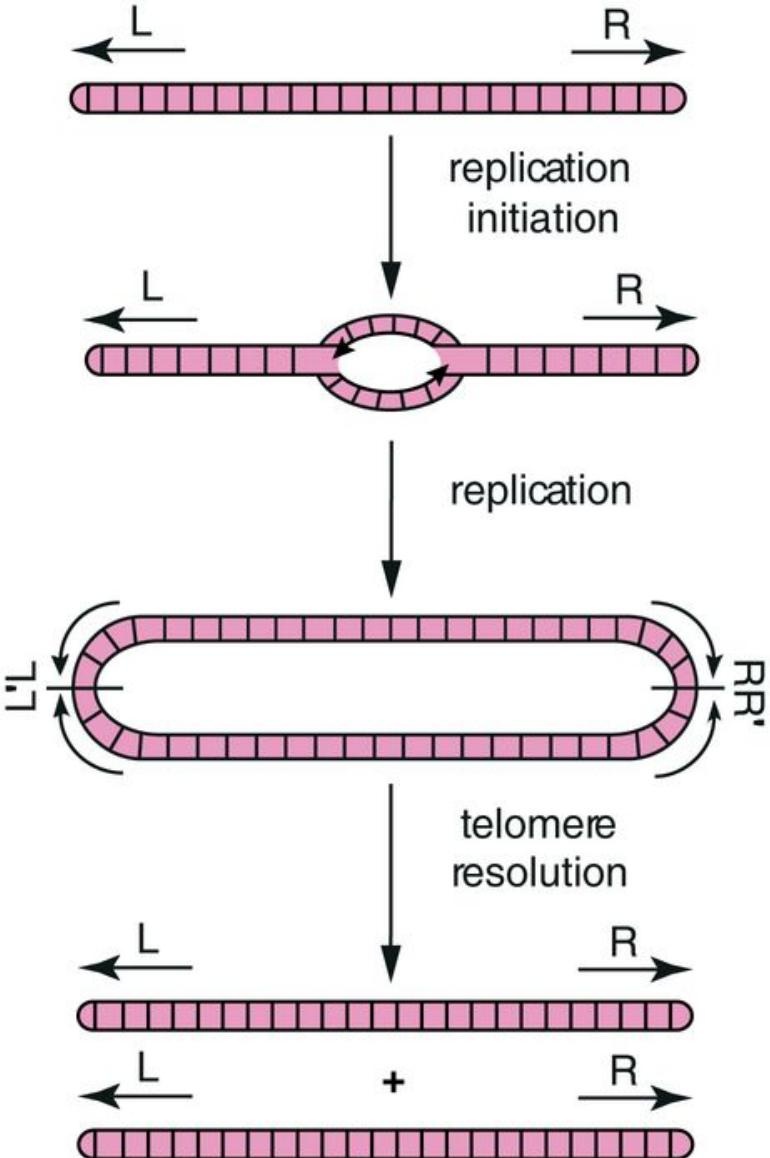


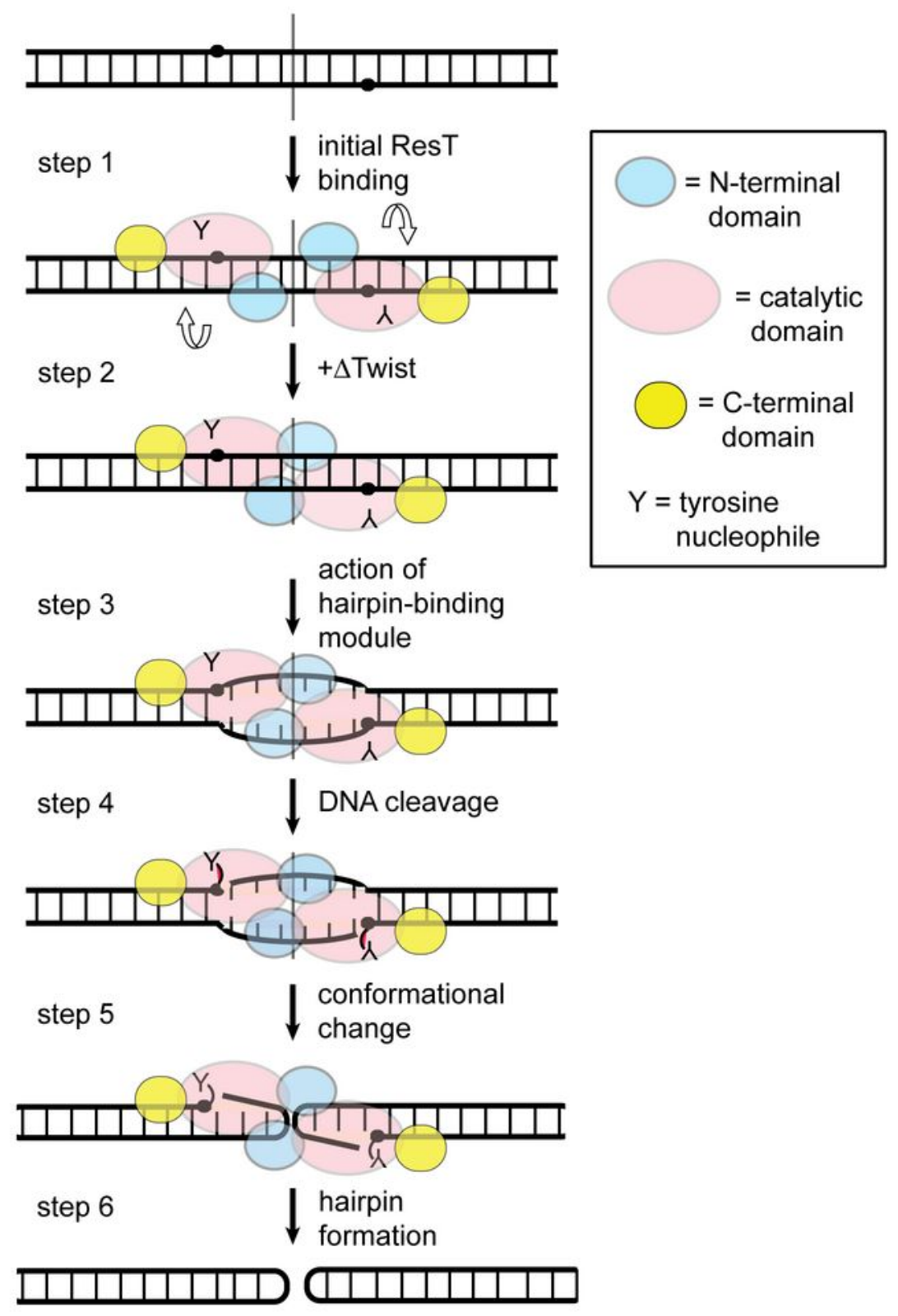




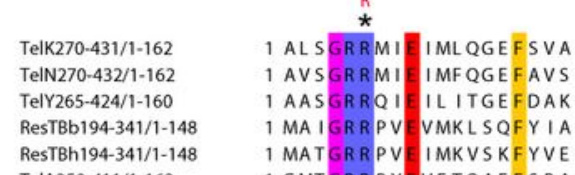

$$
\begin{aligned}
& \text { - II }
\end{aligned}
$$

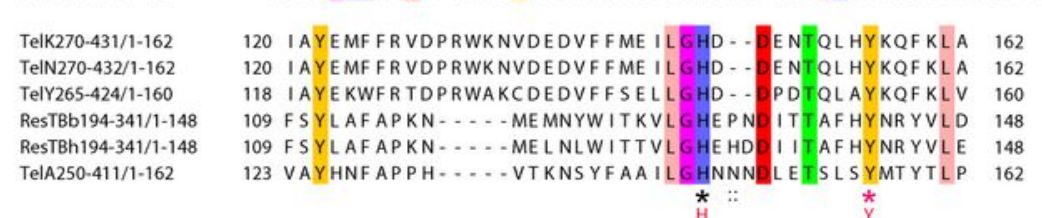


Type Telomere

\section{Sequence}

\section{box 1}

box 3

$2 \quad I p 28-4 R$

$2 \operatorname{Ip} 36 \mathrm{R}$

1 ChromR

$2 \quad$ Ip28-1R

Ip28-1L

2 Ip28-2L, 36L

Ip17L, 28-3L, 28-4L, 21L ATATAATTTTTTATTAGTATAGAGT

2 Ip28-3R

Ip56R, Ip17R

2 Ip38R

$2 \quad \mathrm{Ip} 56 \mathrm{~L}$

$3 \quad$ Ip21R

2 ChromL

$3 \quad$ Ip25L

3

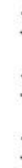

ATATTATTATTACTTAGTATAAATT

ATATAATATTTATTTAGTACAAAGT

AATTTATTATCTTTTAGTATAATAG

AATTTATTATCTTTTAGTATAATGC

AAATTAGTTTTTTTTAGTATAAAGC

TAAATATAATTTAATAGTATAAAAA

TATAAATTTTTAAATAGTATAGTTA

AAATATTTTTTTATTAGTATAGAGA

ATACAATTATTAATTAGTATAGAAA

AATTTACAATTTTTTAGTATAAAAA

TGAAGATAATCTATTAGTATACTAA

TAATAAGAGTTTATTAGTATACTAA
Initial Rate

(fmol/min)

$48.8 \pm 2.5$

$43.0 \pm 7.8$

$41.7 \pm 7.3$

$41.4 \pm 0.9$

$40.4 \pm 7.4$

$39.3 \pm 13.8$

$33.7 \pm 6.1$

$29.2 \pm 6.8$

$28.4 \pm 2.8$

$27.4 \pm 0.1$

$26.5 \pm 5.4$

$26.5 \pm 6.1$

$25.6 \pm 0.4$

$18.3 \pm 3.1$

$13.6 \pm 2.1$

$4.7 \pm 2.2$

$<0.3$

$<0.3$

$<0.3$ 
Chomosomal Common

Right End Extension

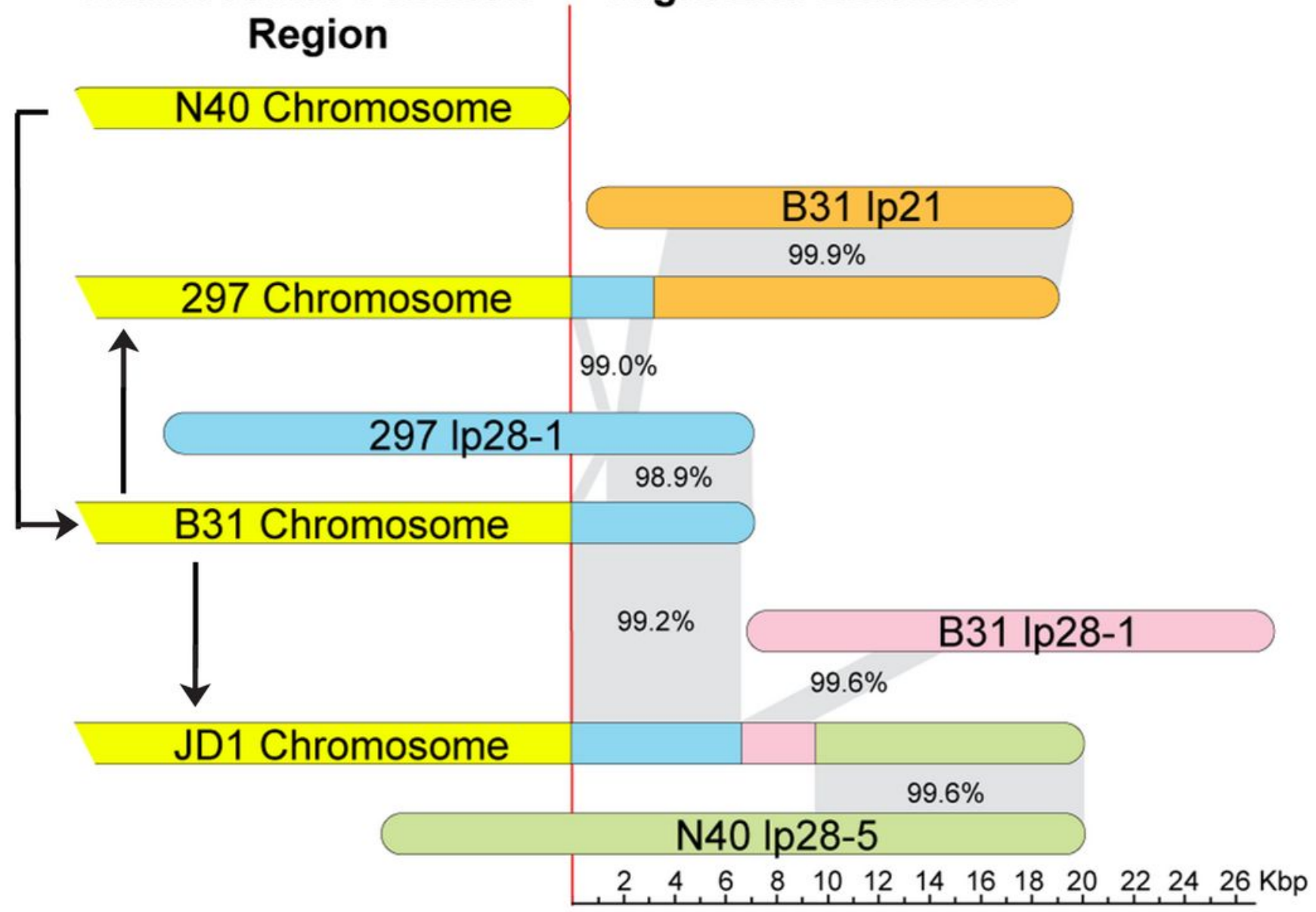




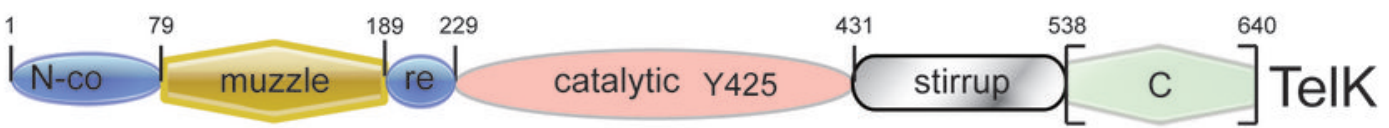

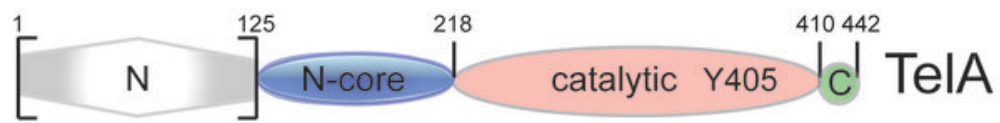

$\mathrm{N}_{\mathrm{N}}^{163} \mathrm{catalyyic}^{13355^{31} \mathrm{C}} \mathrm{Pes}$

B

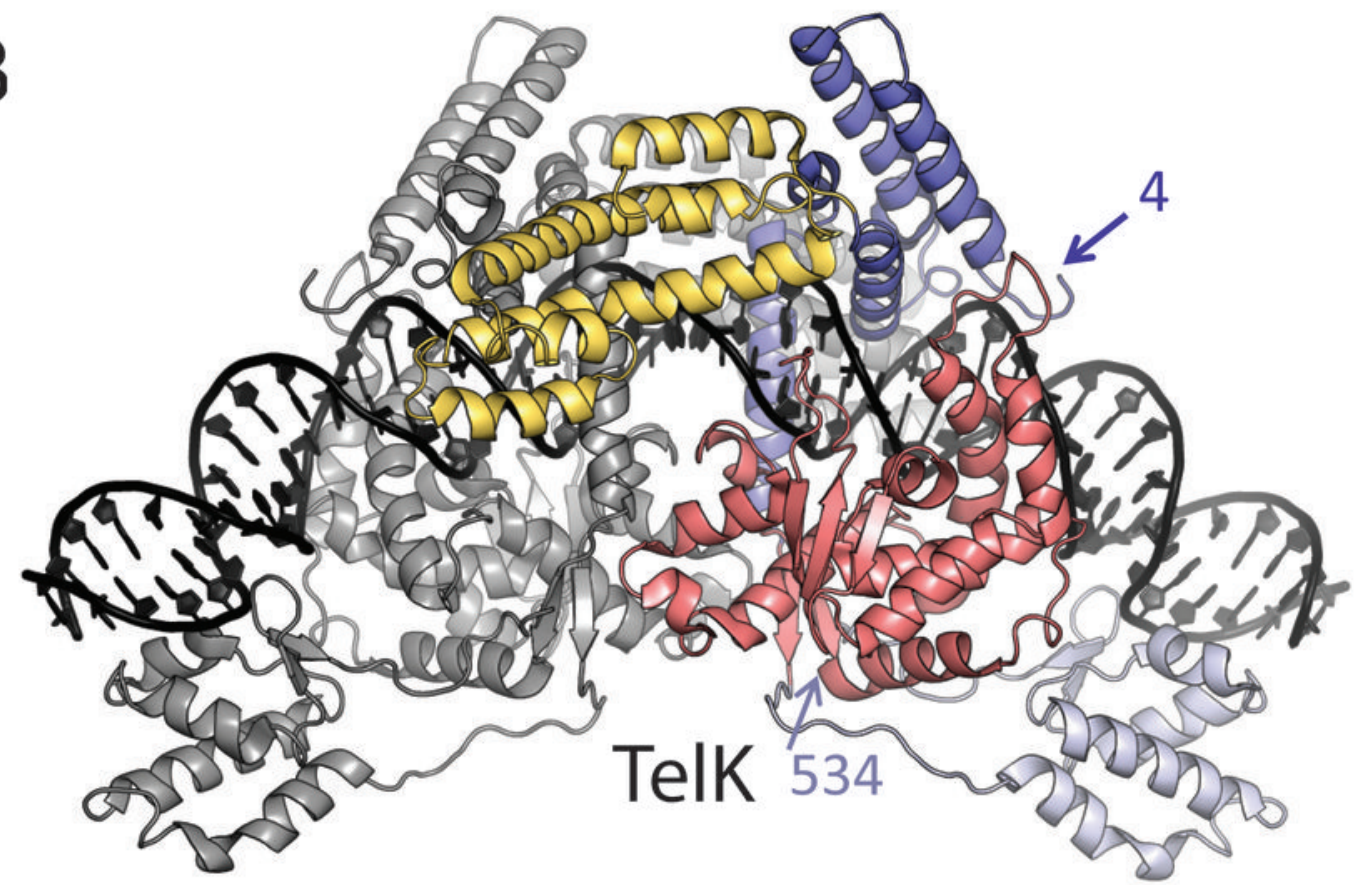

C

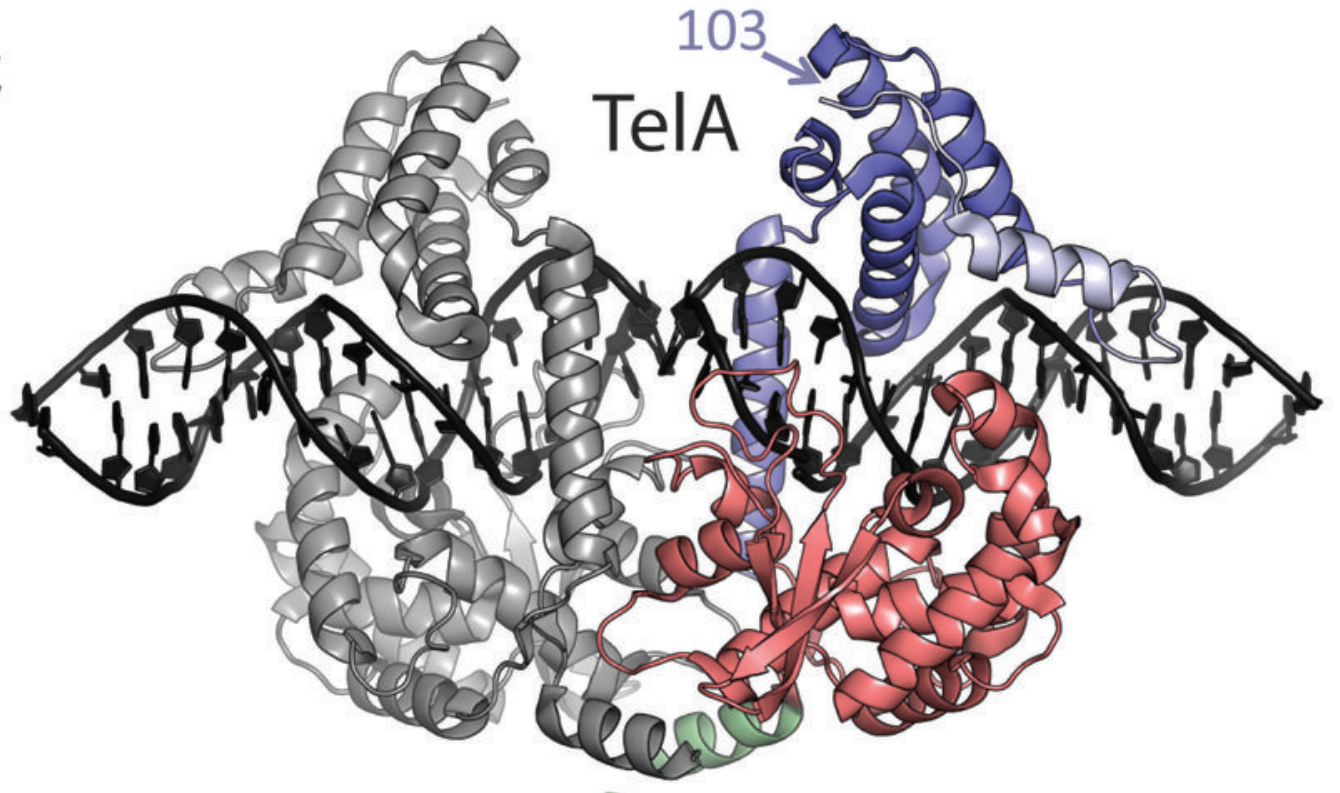

$421 \nearrow$ 


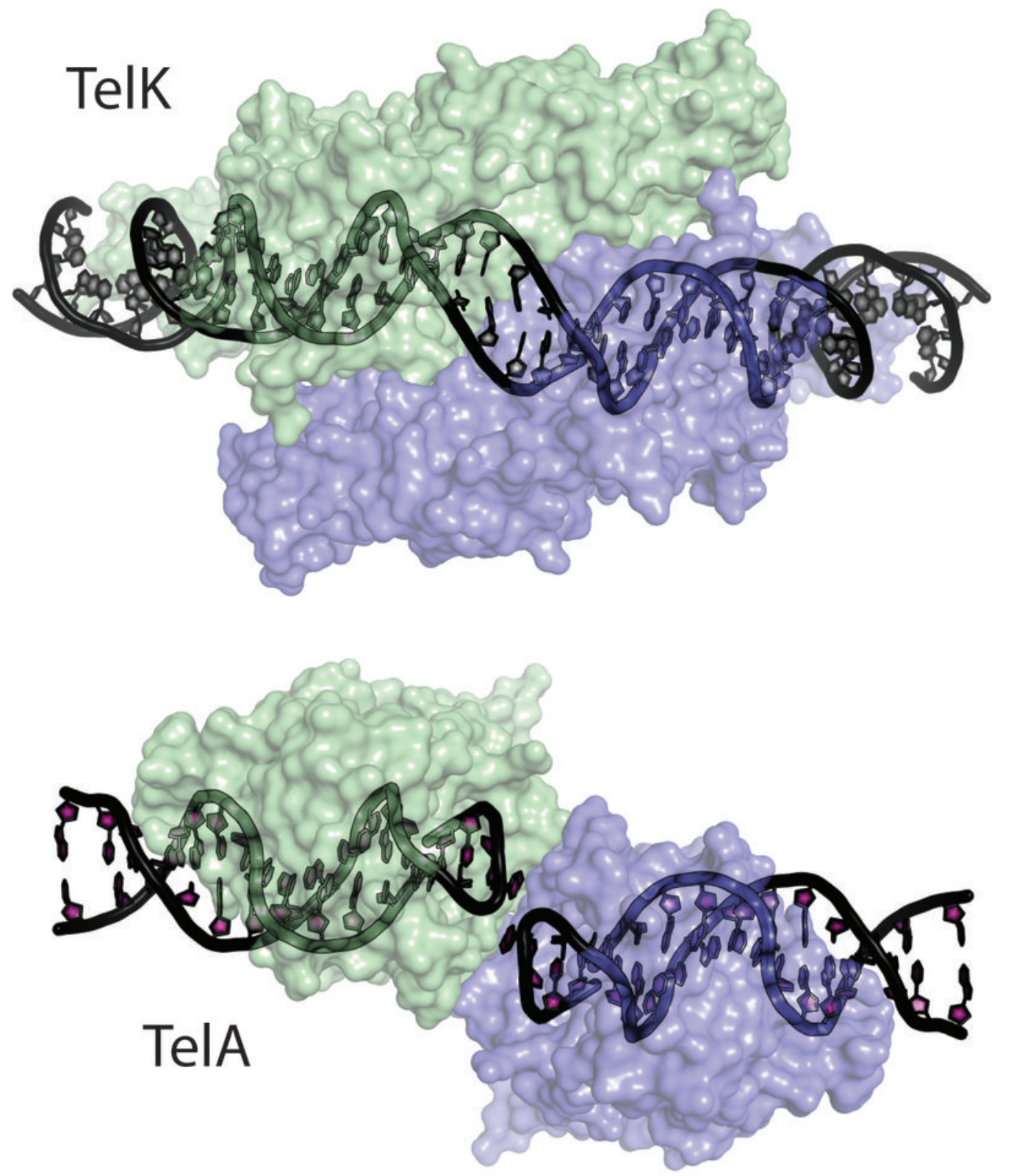

